\title{
Towards a He-buffered laser ablation ion source for collinear laser spectroscopy
}

\section{Tim Ratajczyk $^{1}$ (D) Philipp Bollinger ${ }^{1}$. Tim Lellinger ${ }^{1}$. Victor Varentsov ${ }^{2,3}$. Wilfried Nörtershäuser ${ }^{1}$}

Published online: 20 April 2020

(C) The Author(s) 2020

\begin{abstract}
Laser ablation opens a material-independent method to produce ions from transition metals for laser spectroscopy. To overcome some drawbacks of this process, an ion source is under development at TU Darmstadt. A distinctive feature of this source is that ions are produced via laser ablation in presence of helium buffer gas where they stop and cool in the process of their collisions with the buffer gas atoms and are then extracted by the gas flow into low-pressure conditions through the supersonic nozzle. The compact RF-only funnel ion guide placed on the axis behind the nozzle exit allows for effective extraction of highquality ion beams into a pressure region below $10^{-4}$ mbar. The extraction is realized by using the gas flow trough a supersonic nozzle and an RF-only funnel ion guide, followed by a second nozzle and an RF+DC funnel representing two differential pumping stages. The technical details of this laser ablation ion source are described and the results of the first tests with the RF-only funnel are presented.
\end{abstract}

Keywords Ion source - Laser ablation - RF funnel - Gas dynamic and ion trajectory simulations $\cdot$ Collinear laser spectroscopy

Mathematics Subject Classification (2010) 76-10

This article is part of the Topical Collection on Proceedings of PLATAN 2019, 1st International Conference, Merger of the Poznan Meeting on Lasers and Trapping Devices in Atomic Nuclei Research and the International Conference on Laser Probing, Mainz, Germany 19-24 May 2019

Edited by Krassimira Marinova, Michael Block, Klaus D.A. Wendt and Magdalena Kowalska

Tim Ratajczyk

tratajczyk@ikp.tu-darmstadt.de

1 Institut für Kernphysik, Technische Universität Darmstadt, Deutschland, Germany

2 Facility for Antiproton and Ion Research in Europe (FAIR GmbH), Darmstadt, Germany

3 Institute for Theoretical and Experimental Physics, Moscow, Russia 


\section{Introduction}

Ions for laser spectroscopic investigations can be produced in a variety of ways, reaching from surface ionization on hot materials, laser resonance ionization to ionization in electronproduced plasmas in various ion sources like ECR, Penning, and electron-beam ion sources [1]. A very versatile technique is laser ablation which can be applied for ion production in vacuum from all solid materials and even compound materials. The drawback of this method is that the ions are produced in a plasma at very high temperatures, typically in the order of $10^{5} \mathrm{~K}$ and the ions are released over a $2 \pi$ solid angle into the ablation chamber [2]. Sometimes a low emittance is not needed because the ions do not have to be transported [3], only a few ions are sufficient [4, 5], lower precision tests are performed [6] or other methods like highly time-resolved measurements are used to compensate the emittanceinduced effects afterwards [7].

When the ablation process takes place in the presence of a buffer gas (e.g. helium), the produced ions stop and cool due to numerous collisions with the buffer-gas atoms. Using low-pressure buffer gas simplifies the differential pumping progress but catches a lower fraction of the ablated ions. While this can be sufficient for trap measurements [8] collinear laser spectroscopy profits from a high number of ions per bunch and especially from a lowemittance beam. This can be achieved by ablating the ions in buffer-gas pressures above 10 mbar and extracting them from the laser ablation chamber into low-pressure conditions through a nozzle in a supersonic gas jet as it is realized in other cases where ions are generated or stopped in high-pressure gas cells by other means. Often RFQ's or sextupole ion guides are used to guide the ions in these experiments [9-11]. Here, the extraction of ions into high vacuum is performed with the use of an RF-only ion funnel placed on the axis downstream the nozzle exit. This approach to the ion beam extraction has been proposed in [12] and later it was further developed [13-15] and experimentally proven at ETH Zürich $[16,17]$ and Stanford [18, 19].

Such a system is also of high interest for gas stoppers at in-flight facilities, since it combines stopping, cooling and bunching devices. The only collinear laser spectroscopy that is currently operating at an in-flight facility is the BEam COoler and LAser spectroscopy (BECOLA) setup at NSCL. There, the radioactive isotopes produced in projectilefragmentation reactions and subsequent in-flight separation [20] are thermalized in the NSCL gas stopper [21] and extracted at low energy (typically $30 \mathrm{keV}$ ). The low-energy beam is then transported to the BECOLA RFQ cooler and buncher [22], where it has to be recaptured for cooling and bunching in a low-pressure region before it is finally injected into the collinear laser spectroscopy beam line [23]. Using the compact double funnel system introduced here could simplify such a setup considerably.

In order to equip our collinear beamline COALA (COllinear Apparatus for Laser spectroscopy and Applied science) at TU Darmstadt [24-26] with a versatile ion source that will also serve the LASPEC experiment at FAIR [27], we are currently developing a laserablation ion source. The source is designed to reduce necessary DC fields to a minimum, but maintaining a high transport efficiency by He gas flow. It is the third design version of the laser ablation ion source with the RF-only funnel. The first design [14] used a target in form of a thin rod placed into the central tube of the annular nozzle while the laser beam entered through holes in the electrodes. The gas flow rate through the relatively large cross section of the annular nozzle throat demanded higher pumping speed in the RF-funnel chamber. In the second design $[16,17]$ the laser had to be focused and aligned through the whole apparatus and through the nozzle reducing the freedom in the target design and limiting the reduction 


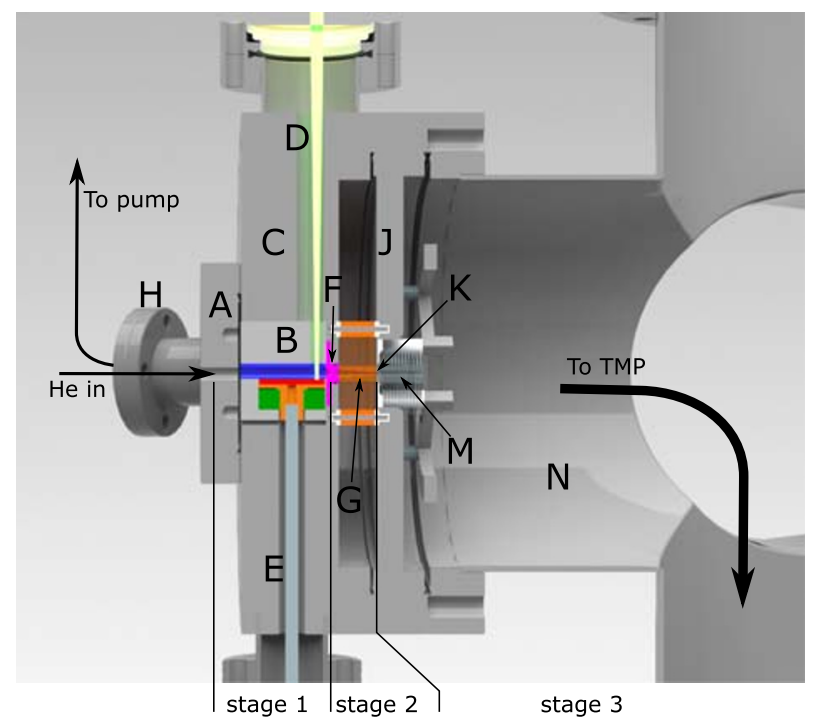

Fig. 1 Overview over the setup with laser beam (D), ablation stage (B), funnel stage (F,G) and detection stage $(\mathrm{K}, \mathrm{M}$ in $\mathrm{N})$. Details are provided in the text

of the nozzle diameter. In the third design presented in this work the laser ablation is realized orthogonal to the nozzle and funnel axis allowing the laser ablation and ion extraction to be designed independently. Moreover, this ion source has an additional miniature RF+DC funnel (placed behind the RF-only funnel), through which the ablated ions are extracted smoothly into high vacuum. The time-length of the created ion beam pulse is solely determined by the dynamics of the buffer-gas flow. The second funnel is also designed to use an additional DC voltage applied to its last electrode to accumulate ions in a small part of the funnel and then extract them in form of a short, intense and high-quality bunch. Here, we describe the technical details of the new laser ablation ion source and the results of the first tests with the RF-only funnel.

\section{Setup}

The design of the ion source should provide cooling and efficient transport of the ablated ions into vacuum using a buffer carrier gas. To maintain vacuum conditions below $10^{-4}$ mbar at the output of the source a turbo molecular pump should be used and therefore a vacuum chamber cross of $150 \mathrm{~mm}$ diameter (CF $150 \mathrm{cross}$ ) is the central piece. The setup consists of three different pressure stages. Those three stages are each mounted onto a vacuum flange to ensure sealing between them as shown in Fig. 1. At the first stage the laser ablation process takes place and produces the ions under helium ambience. The helium is leaked in with a valve through the entrance flange (A) into the ablation unit (B). This unit is located inside the 150-mm main flange (C) with a bore for the laser (D) and a bore for a rotation feedthrough $(\mathrm{E})$. The first and the second pressure stages are connected by a converging-diverging conical nozzle $(\mathrm{F})$. A more detailed drawing of the inner section is shown in Fig. 2. At the second stage the ions are transported by the gas stream and are confined on the central axis by an RF-only funnel $(\mathrm{G})$. Pumping of this chamber is realized 

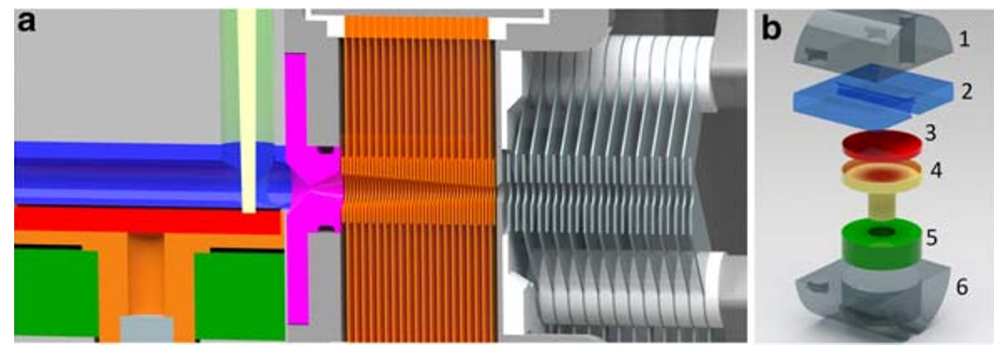

Fig. 2 Detailed layout of the setup: a Technical drawing of the ion source with ablation unit (color code as in the figure to the right): $1^{\text {st }}$ nozzle (magenta), funnel section (orange), and $2^{\text {nd }}$ RF funnel (gray) with insulators (white). b Exploded view of the ablation unit: (1) top part with laser bore, (2) middle part with 5-mm ablation chamber, (3) 1-inch sputter target, (4) target holder, (5) ceramic bearing, (6) bottom part

through a tube that connects the second stage with the vacuum flange $(\mathrm{H})$ affiliated to a dry pump. The third stage is delimited by the separation flange $(\mathrm{J})$ that has a $1 \mathrm{~mm}$ tapered hole in the center forming the second nozzle $(\mathrm{K})$. On the other side of this flange, a second RF funnel (M) can be mounted. Flanges (C) and (J) are mounted in series on one arm of a vacuum cross (N) that is equipped with a 900-1/s turbo-molecular-pump (TMP) to evacuate the remaining gas flow.

\subsection{First stage - laser ablation}

The first stage consists mainly of the ablation unit as shown in Fig. 2b. This ablation unit is manufactured as an exchangeable part inside the main flange (see Fig. 1) to allow us to replace the target by opening only the entrance flange and removing the ablation unit. This also enables us to redesign this part without changing the rest of the apparatus, for example, to put in targets of a different shape.

The 5-mm bore in the middle part (2) of the ablation unit is the ablation chamber through which the carrier gas streams towards the ablation region and the extraction nozzle. The beam of the laser is directed onto the target material underneath the ablation chamber through a 5-mm bore that is drilled in the top part (1) of the ablation unit. The 5-mm diameter was a compromise to keep a reasonable velocity of the carrier gas and provide sufficient space to adjust the laser beam. This hole expands in the main flange to a $10-\mathrm{mm}$ bore connected to a window flange, through which the laser light enters the vacuum chamber.

The target (3) is mounted in the bottom part (6) of the ablation unit. It is a 1-inch sputter target because many different elements are commercially available in high purity in this geometry. It also allows to rotate the target and hence to change the ablation spot if necessary without opening the vacuum chamber. If some elements are not available in the 1-inch sputter format a stainless steel holder of that shape can be produced to fit in smaller samples or to equip the holder with several different elements. The target is mounted on a targetholder (4) that can be connected to the shaft of a rotational feedthrough, being equipped with a stepper motor to either change to a new ablation spot or switch between different elements that might be mounted on the target. To reduce friction, a ceramic bearing (5) fixates the target holder in the lubricant free environment of the vacuum chamber. In Fig. 2a details of the system are shown as a drawing of the technical implementation. One can see on the left in grey the top and bottom part of the ablation unit and in blue the middle part with the ablation chamber and in red the target. 
The laser (Lumibird Centurion+) used for ablation is a frequency-doubled, diodepumped, Q-switched Nd:YAG with up to $25 \mathrm{~mJ}$ at $532 \mathrm{~nm}, 10$-ns pulse length and a repetition rate of up to $100 \mathrm{~Hz}$. The output energy can be controlled via an on-board attenuator from $0 \mathrm{~mJ}$ to $25 \mathrm{~mJ}$. The laser beam is then widened by a telescope to about $6 \mathrm{~mm}$ to be finally focused by a $175-\mathrm{mm}$ lens onto the target surface. The size of the ablation crater was measured by a microscope to be $600-\mu \mathrm{m}$ diameter. To facilitate alignment of the ablation laser onto the target, the pulsed beam is superimposed with a 532-nm laser beam from a commercial diode laser. The target can be illuminated by a ring of LEDs through the same window flange as the laser enters. That allows to observe the beam spot on the target and to adjust the beam position by a camera, positioned behind the last mirror used to steer the laser beam into the chamber. During measurement the camera can be equipped with a 532$\mathrm{nm}$ absorption filter to monitor the visible degradation of the target. High-purity He N50 is used as carrier gas. It comes in a 10-1, 200-bar bottle, and the pressure gets reduced to 1 bar prior to being fed into the system by an all-metal gas-dosing valve.

\subsection{Second stage - first RF-funnel}

The first and the second stage are connected through a converging diverging nozzle with a throat diameter of $0.3 \mathrm{~mm}$ and an angle of $45^{\circ}$ on the subsonic converging and $26.5^{\circ}$ on the supersonic diverging side and is colored magenta in Fig. 2a. The nozzle establishes a supersonic jet into the second stage able to transport the ions only by the gas flow. Connected to the second stage a $40-\mathrm{m}^{3} / \mathrm{h}$ dry pump (Leybold Ecodry 40 plus) is used for evacuation. Main design parameters of the nozzles and funnels are presented in Table 1. A schematic view of the double-funnel system combined with results of the gas-dynamic simulation for the helium velocity flow-field are shown in Fig. 3. This gas-dynamic simulation is based on the solution of a full system of time dependent Navier-Stokes equations for multicomponent gas mixtures and is described in detail in [28]. The ablated ions move under a combined effect of the gas dynamic and electric field. That is why the results of the gas-dynamic simulations (flow fields of the buffer gas velocity, density and temperature) were then incorporated into a Monte Carlo ion trajectory simulations code, for which the electric fields were simulated

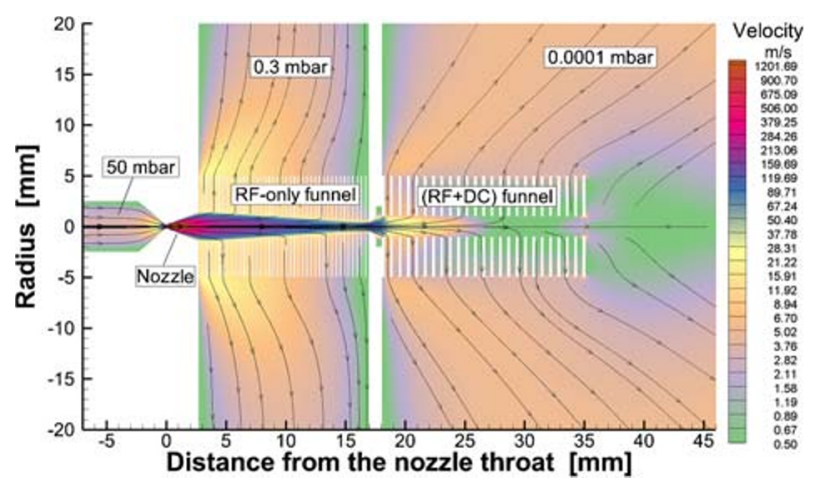

Fig. 3 Schematic view of the double-funnel system combined with results of the gas dynamic simulation for the helium velocity flow field. Black arrowed lines show the gas flow direction. The $1^{\text {st }}$ and $2^{\text {nd }}$ nozzle throats diameters are $0.3 \mathrm{~mm}$ and $1.0 \mathrm{~mm}$, correspondingly. The pressure in the ablation chamber is $50 \mathrm{mbar}$ resulting in a pressure of $0.3 \mathrm{mbar}$ at the chamber of the first and $10^{-4} \mathrm{mbar}$ at the chamber of the second funnel 
Table 1 Main design parameters of the nozzles and funnels

$\begin{array}{lll}\text { Geometry of the nozzles } & & \\ \text { Nozzle } & 1^{\text {st }} & 2^{\text {nd }} \\ \text { Half-angle of converging cone } & 45.0^{\circ} & - \\ \text { Half-angle of diverging cone } & 26.5^{\circ} & 45.0^{\circ} \\ \text { Length of diverging cone } & 2.7 \mathrm{~mm} & 0.5 \mathrm{~mm} \\ \text { Throat diameter } & 0.3 \mathrm{~mm} & 1.0 \mathrm{~mm} \\ \text { Exit diameter } & 3.0 \mathrm{~mm} & 2.0 \mathrm{~mm}\end{array}$

Main design parameter of the funnels

\begin{tabular}{lll} 
Funnel & $1^{\text {st }}($ RF-only $)$ & $2^{\text {nd }}(R F+D C)$ \\
Entrance aperture diameter & $3.0 \mathrm{~mm}$ & $2.0 \mathrm{~mm}$ \\
Exit aperture diameter & $1.0 \mathrm{~mm}$ & $2.0 \mathrm{~mm}$ \\
Electrode thickness & $0.1 \mathrm{~mm}$ & $0.2 \mathrm{~mm}$ \\
Inter-electrode spacing & $0.25 \mathrm{~mm}$ & $0.5 \mathrm{~mm}$ \\
Number of electrodes & 41 & 25 \\
\hline
\end{tabular}
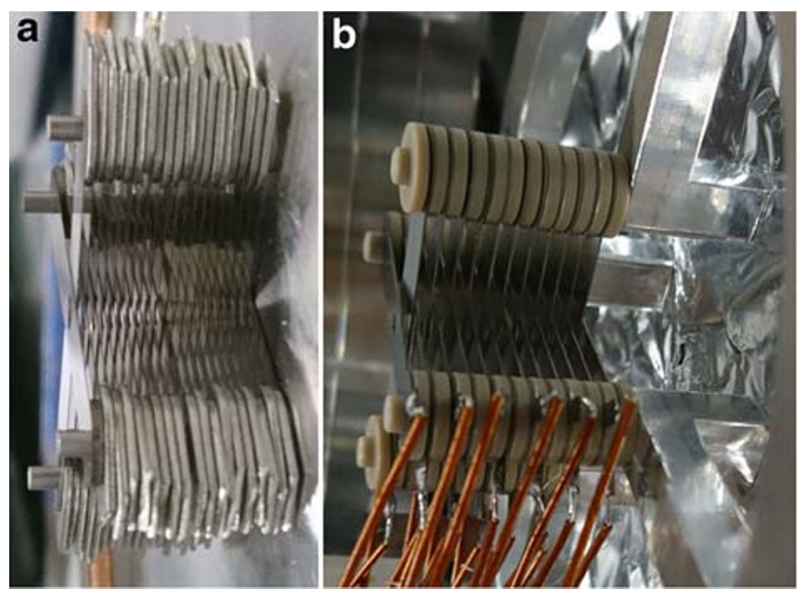

Fig. 4 Close-up pictures of the two funnels: a RF-only funnel before soldered with connection cables. One can see the funnel-like structure of the holes in the electrodes. The rods and spacers are all metal as no DC field is required. b RF+DC funnel with DC gradient. All electrodes are connected separately and isolated with PEEK (Polyether ether ketone) spacers and also PEEK rods. The aluminum cage is used to mount the funnel onto the flange

with the use of SIMION 8.1. Similar simulations and their results are described elsewhere (e.g. see [13-15]).

Ions are confined inside the RF-only funnel (colored orange in Fig. 2a) by an alternating RF field with a frequency between $1 \mathrm{MHz}$ and $10 \mathrm{MHz}$ that produces an effective average potential with a minimum on the central axis. This alternating RF-field is provided via thin electrodes which have holes in the middle with the diameter decreasing from $3 \mathrm{~mm}$ to $1 \mathrm{~mm}$, so that the whole system acts as a funnel for the ions. The electrodes have a thickness of $0.1 \mathrm{~mm}$ and have a surface-to-surface separation of $0.25 \mathrm{~mm}$. The complete funnel stack is shown in Fig. 4. Due to the RF-only design, all electrodes of the same phase can be 
connected using conducting metal spacers. Hence, the electrodes are mounted on a $2 \mathrm{~mm}$ steel rod that is isolated to the vacuum chamber by PEEK insulators and are spaced by cable shoes of 0.6-mm thickness. The electrodes of each phase are soldered together to ensure reliable electric connection. Outside of the funnel core the purity of the helium gas does not influence the ions anymore and therefore degasing of the soldering material should not be an issue.

\subsection{Third stage - second RF-funnel and detection}

The first and the second funnel are connected through a miniature diverging nozzle with $45^{\circ}$ angle and a $1 \mathrm{~mm}$ throat diameter (see Table 1), mounted in the center of the separation flange. The ions enter into the CF- 150 cross that is equipped with a $900-1 / \mathrm{s}$ turbo-pump to maintain a background pressure below $10^{-4}$ mbar. This stage is already designed in a way that allows for mounting the second RF funnel of which a schematic view is shown in Fig. 2a and a photo in Fig. 4b. This RF funnel consists of 25 electrodes of $0.2-\mathrm{mm}$ thick stainless steel. In contrast to the RF-only funnel this RF funnel must be used with a DC gradient to drag the ions because the low-density gas flow here is insufficient for ion transport. For this reason all electrodes need to be set at different potentials and have to be electrically isolated from each other and individually connected. This is realized by stacking the electrodes on PEEK rods and space them by PEEK spacers. Each electrode is soldered to a single capton wire and all 25 wires are connected to a D-sub 25 vacuum feedthrough. This way all the electronics from the RF generation and the resistor chain for the DC gradient can be placed outside of the vacuum chamber, allowing for heat dissipation and easy access of the electric components. The RF signal is coupled in via capacitors.

For the test of the extraction efficiency of the RF-only funnel, the second RF funnel was not installed and the ions were detected about $3 \mathrm{~cm}$ behind the second nozzle at a metal sheet used as a simple Faraday cup. This detector has no means to suppress electron emission after impact, which might lead to a slight overestimation of the ion current, since the emitted electrons also contribute to the positive current measured at the plate. With an acceleration voltage of $-100 \mathrm{~V}$ this effect should be less than a factor of two. The current is then amplified by a trans-impedance amplifier to a voltage signal that is measured by an oscilloscope at its 1-M $\Omega$ resistor. The trans-impedance amplifier has a fall time of $220 \mu \mathrm{s}$, which is considerably faster than the expected ion signal length of about a ms.

\subsection{RF generation}

To provide the RF at the funnel electrodes in the range from $1 \mathrm{MHz}$ to $10 \mathrm{MHz}$ and from $0 \mathrm{~V}_{\mathrm{pp}}$ to $40 \mathrm{~V}_{\mathrm{pp}}$, an RF circuit was built. The basic sine signal can be created by an RF signal generator (Tektronix AFG 3102) and is amplified with a linear power amplifier (Amplifier from Hong Kong, 2 - $30 \mathrm{MHz} 50 \mathrm{~W}$ linear broadband amplifier). The signal after the amplification is still an unbalanced sine signal that needs to be split into two $180^{\circ}$ phase-shifted signals that alternate around $0 \mathrm{~V}$. This is realised with a balanced-unbalanced transformer (balun) that has a ground connection in the center of the second coil shown in Fig. 5(B1) with the different phases in green and red. This transformer is also used to increase the voltage amplitude by having 4 times as many windings on the secondary coil. This steps the voltage up by a factor of 4 and the impedance will be transformed by a factor of 16 [29]. To make this transformer universal for the frequency range between 1 and $10 \mathrm{MHz}$ and independent of the design of the funnel electrodes and the connections, the balun is directly connected to an $800-\Omega$ power resistor which is transformed to $50 \Omega$ seen from the amplifier. 
Fig. 5 Schematic and picture of the RF-system. Top: the schematic of the whole system is shown with the RF-generator $(\mathrm{G})$, the amplifier (A), the BNC connector for the input signal (B2), the balun transformer (B1) with a transformation ratio of $1: 4$ and a GND connection at the center of the second coil, the power resistor $(\mathrm{R})$, the resonance coil (C), the two control connectors (Cont1, Cont2), the D-Sub connector (D1), the vacuum feedthrough connection (D2) and the funnel electrodes which mainly act as capacity (E). Bottom: the actual PCB board is shown with the corresponding components from inside the red-dashed rectangle, but rotated by 90 degree clockwise compared to the schematic
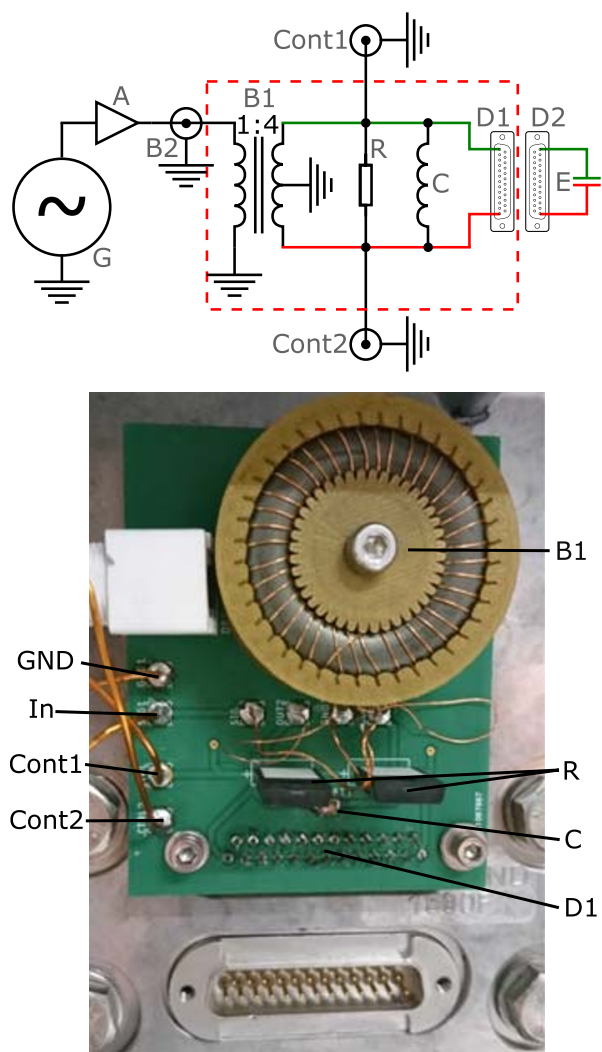

This way it is always assured that the amplifier has to drive an impedance close to $50 \Omega$ over a wide range of frequencies. A ferrite material (Amidon FT 114-77) was used as core of the balun.

The funnel electrodes act as a capacity connected parallel to the resistor $(\mathrm{R})$. This changes the impedance of the secondary coil of the balun, which can be compensated by an additional coil (C). This way the coil (C) and the Funnel electrodes (E) form a parallel resonant circuit that acts as a high impedance compared to the 800- $\Omega$ resistor at resonance frequency. Changing the coil $(\mathrm{C})$ or adding additional capacity between the two phases will allow for frequency tuning.

The signal generator, the amplifier and the electronics in the red-dashed rectangle in Fig. 5a are placed outside the vacuum to allow heat dissipation and access without breaking the vacuum. The connection is realised through the D-sub 25 feedthrough and capton cables to the funnel electrodes. The second connector seen in Fig. 5 can be used to feed all 25 electrode connections for the second funnel (see Section 2.3) separately into the vacuum chamber, while all electronic components are accessible outside.

\section{Measurements}

A stainless steel target was used for first tests because it contains mainly iron which is a transition metal and can be produced and handled without further safety concerns. The 


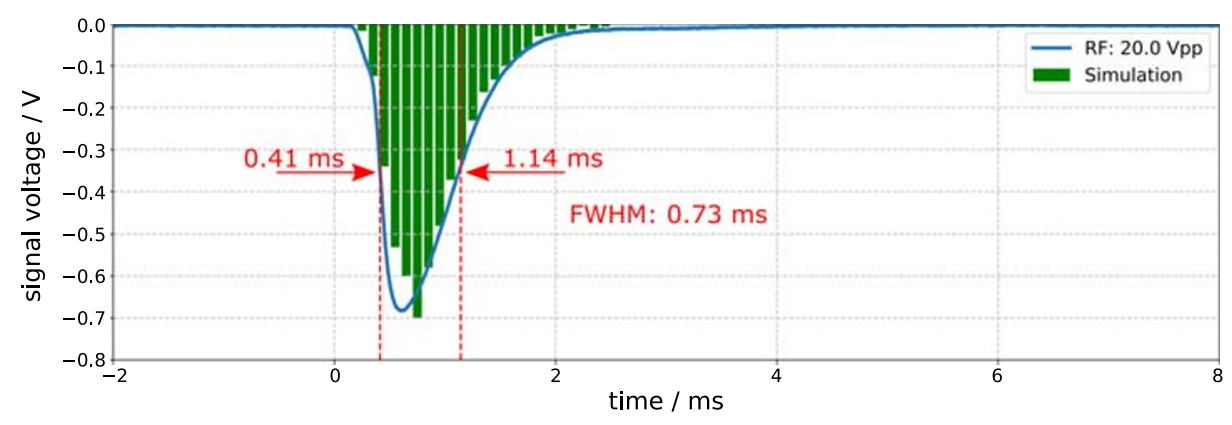

Fig. 6 Typical ion signal, average of eight consecutive ion pulses. The FWHM is of the order of a ms

number of ions was determined from the integrated voltage signal taking into account the amplification factor of the 220-M $\Omega$ resistor of the trans-impedance amplifier. This amplifier also has the property that it changes the sign of the signal, so that the positive ions generate a negative signal.

\subsection{Typical signal}

A typical signal is shown in Fig. 6. The data acquisition was triggered by the signal for the Q-switch of the pulsed laser, which is marked as zero on the horizontal axis. The amplifier has a noise of about $1 \mathrm{mV}$ on the signal that was not correlated with ion pulses. To suppress this noise, eight consecutive ion pulses were averaged directly on the oscilloscope. The term "ion signal" will be used in the following for such an averaged signal. To ensure full signal registration, data was taken from $2 \mathrm{~ms}$ before the trigger until $8 \mathrm{~ms}$ after the trigger, covering a total period of $10 \mathrm{~ms}$. The green histogram is the result of the simulation of the ions transport time. To get comparable values the ablated ions were simulated as an ion cloud stopped in the center of the 5-mm tube in the ablation chamber at the distance of $2.5 \mathrm{~mm}$ from the nozzle entrance in accordance with the distance of the laser ablation spot. This way not only the transport through the funnel but also the transport to the nozzle is taken into account in the simulation. The result was then scaled with the height of the measured signal. The shape of the simulated $20 \mathrm{~V}_{\mathrm{pp}}$ signal and its time position come directly from the simulation and are in good agreement with the measured signal.

\subsection{Variation of the RF-amplitude}

To test the transmission efficiency through the funnel as a function of the RF-amplitude, a constant pressure of 50 mbar was maintained in the ablation section. The RF-amplitude was then step-wise increased from 0 to $50 \mathrm{~V}_{\mathrm{pp}}$. For each data point 20 ion pulse measurements were taken to measure how the signal varies. In Fig. 7 the observed ion signal is shown as a function of the RF-amplitude. The signal value for each RF-voltage amplitude represents the mean value of 20 measurements and the error bars represent the corresponding standard deviation. Systematic uncertainties are not taken into account. For low RF voltages the signal is constant and starts to rise strongly from $10 \mathrm{~V}_{\mathrm{pp}}$ up to a maximum between 25 and $35 \mathrm{~V}_{\mathrm{pp}}$. Then the signal decreases again for higher amplitudes. This overall behavior 


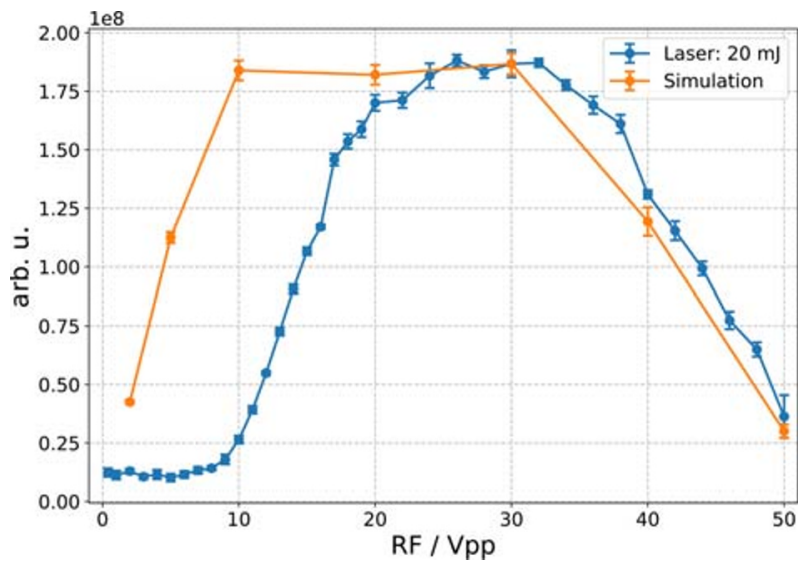

Fig. 7 Ion signal as a function of the RF-amplitude
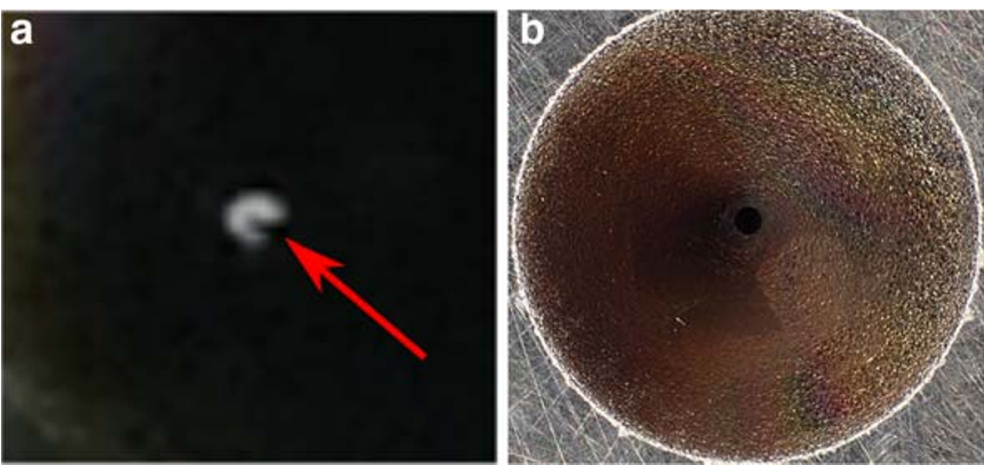

Fig. 8 a Picture of the nozzle after the measurements. The arrow points to a place where something is blocking a part of the nozzle throat. Picture $\mathbf{b}$ was taken after cleaning the nozzle with a better microscope

is in agreement with the simulations which are shown scaled in amplitude according to the $30 \mathrm{~V}_{\mathrm{pp}}$ value. Nevertheless, the simulation predicts an earlier rise of the signal what might be a hint of not yet found systematic influences by the the system of which one might be space charge, as it is not considered in the simulations.

Opening the vacuum chamber after the first test measurements presented here, we had to note that the throat diameter was reduced by a part of ablated material as shown in Fig. 8. The reduced diameter has no influence on the efficiency of stopping the ions within the 5-mm ablation chamber but has an impact on the gas dynamics. Therefore, further investigations are required. Nevertheless, the principle functionality of the ion source was already demonstrated in these measurements.

\subsection{Effect of the gas-stagnation pressure on the ion extraction}

In the measurement shown in Fig. 9, the extraction of ions has been investigated as a function of the pressure in the ablation chamber. The RF amplitude at the funnel was held 


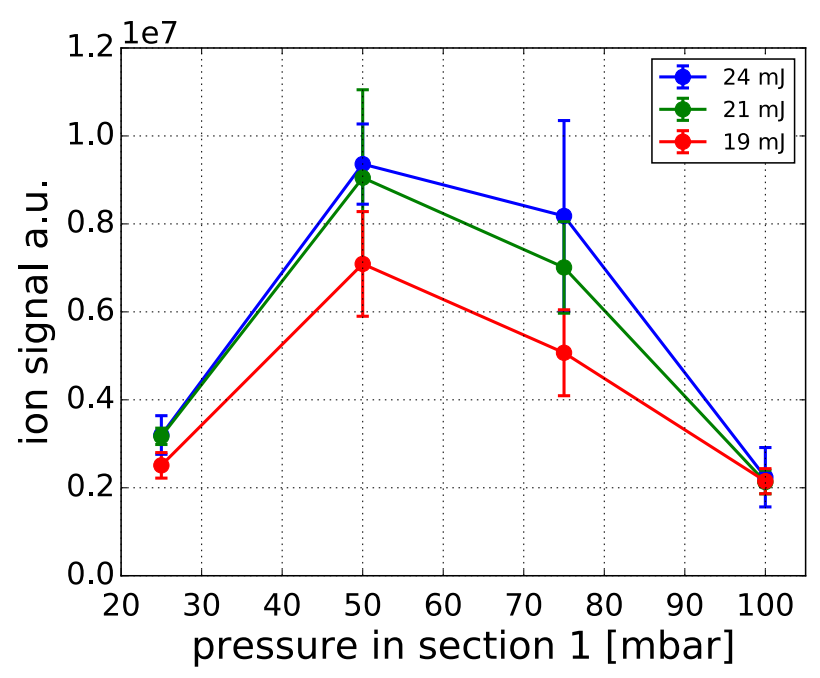

Fig. 9 Relative extraction efficiencies of ions through the first funnel at different pressures in the ablation chamber. The highest signal at 50 mbar supports the assumption that at this pressure the ions are being stopped closest to the center of the ablation chamber, leading to the most efficient extraction through the first nozzle

constant at $20 \mathrm{~V}_{\mathrm{pp}}$ and at a frequency of $5 \mathrm{MHz}$, which should lead to high transmission through the system of both RF funnels according to Fig. 7. The ion signal was measured for $19,21,24 \mathrm{~mJ}$ at each pressure and the data point represents the average ion count and the error bar the standard deviation. The measurement shows that the extraction from the ablation spot to the nozzle throat is most efficient at 50 mbar. It also shows that this is true for the three laser energies that were tested.

\section{Conclusion and outlook}

We presented the setup and first operation of the laser ablation ion source developed at TU Darmstadt. The electronics for RF generation produce RF signals from 2 to $10 \mathrm{MHz}$ with amplitudes up to $50 \mathrm{~V}_{\mathrm{pp}}$ and are hence a versatile tool to investigate the parameters of this ion source.

The ion source is able to produce ions from laser ablation of transition metal targets and extract them into a vacuum below $10^{-4}$ mbar. The ions are already in a bunched shape of about 1-ms pulse length. The extraction efficiency of the system correlates with the RF amplitude of the extraction funnel and shows trapping effects for RF amplitudes above $35 \mathrm{~V}_{\mathrm{pp}}$. For further analysis of the system more parameters and the impact of effects like space charge have to be investigated.

Acknowledgements Open Access funding provided by Projekt DEAL. We acknowledge financial support from the German Federal Ministry for Education and Research (BMBF) under Contract No. 05P19RDFN1 and from HIC for FAIR. T.R. acknowledges support from HGS-HIRe. 
Open Access This article is licensed under a Creative Commons Attribution 4.0 International License, which permits use, sharing, adaptation, distribution and reproduction in any medium or format, as long as you give appropriate credit to the original author(s) and the source, provide a link to the Creative Commons licence, and indicate if changes were made. The images or other third party material in this article are included in the article's Creative Commons licence, unless indicated otherwise in a credit line to the material. If material is not included in the article's Creative Commons licence and your intended use is not permitted by statutory regulation or exceeds the permitted use, you will need to obtain permission directly from the copyright holder. To view a copy of this licence, visit http://creativecommonshorg/licenses/by/4.0/.

\section{References}

1. Wolf, B. Handbook of Ion Sources, 1st edn. CRC Press, Boca Raton (2017)

2. Stafe, M., Marcu, A., Puscas, N.N.: Pulsed Laser Ablation of Solids, vol. 53. Springer, Berlin (2014)

3. Zimmermann, K., Okhapkin, M.V., Herrera-Sancho, O.A., Peik, E.: Laser ablation loading of a radiofrequency ion trap. Appl. Phys. B 107(4), 883 (2012). https://doi.org/10.1007/s00340-012-4884-1

4. Sailer, T.: A laser ion source for the ALPHATRAP experiment

5. Sturm, S., Arapoglou, I., Egl, A., Höcker, M., Kraemer, S., Sailer, T., Tu, B., Weigel, A., Wolf, R., López-Urrutia, J.C., Blaum, K.: The ALPHATRAP experiment. Europ. Phys. J. Special Topics 227(13), 1425 (2019). https://doi.org/10.1140/epjst/e2018-800225-2

6. Renth, L., Imgram, P., Krämer, J., König, K., Lellinger, T., Maaß, B., Müller, P., Ratajczyk, T., Nörtershäuser, W.: First experiments with a laser ablation source at the COALA setup, PLATAN proceedings (2020)

7. Garcia Ruiz, R.F., Vernon, A.R., Binnersley, C.L., Sahoo, B.K., Bissell, M., Billowes, J., Cocolios, T.E., Gins, W., de Groote, R.P., Flanagan, K.T., Koszorus, A., Lynch, K.M., Neyens, G., Ricketts, C.M., Wendt, K.D.A., Wilkins, S.G., Yang, X.F.: High-precision multiphoton ionization of accelerated laserablated species. Phys. Rev. X, 8(4). https://doi.org/10.1103/PhysRevX.8.041005 (2018)

8. Schneider, F., Beyer, T., Blaum, K., Block, M., Chenmarev, S., Dorrer, H., Düllmann, C.E., Eberhardt, K., Eibach, M., Eliseev, S., Grund, J., Köster, U., Nagy, S., Novikov, Y.N., Renisch, D., Türler, A., Wendt, K.: Preparatory studies for a high-precision penning-trap measurement of the ${ }^{163}$ Ho electron capture Q-value. Europ. Phys. J. A 51(7), 447 (2015). https://doi.org/10.1140/epja/i2015-15089-8

9. Äystö, J.: Development and applications of the IGISOL technique. Nucl. Phys. A 693(1-2), 477 (2001). https://doi.org/10.1016/S0375-9474(01)00923-X

10. Wense, L., Seiferle, B., Laatiaoui, M., Thirolf, P.G.: The extraction of ${ }^{229} \mathrm{Th}^{3+}$ from a buffer-gas stopping cell. Nucl. Instrum. Methods Phys. Res., Sect. B 376, 260 (2016). https://doi.org/10.1016/j.nimb.2015.12.049

11. Savard, G., Levand, A.F., Zabransky, B.J.: The CARIBU gas catcher. Nucl. Instrum. Methods Phys. Res., Sect. B 376, 246 (2016). https://doi.org/10.1016/j.nimb.2016.02.050

12. Varentsov, V.: A new approach to the extraction system design. https://doi.org/10.13140/RG.2.2.30119. 55200

13. Varentsov, V., Wada, M.: Computer experiments on ion beam cooling and guiding in fair-wind gas cell and extraction rf-funnel system. Nucl. Instrum. Methods Phys. Res., Sect. A 532(1-2), 210 (2004). https://doi.org/10.1016/j.nima.2004.06.078

14. Varentsov, V.: Focused ion beam source of a new type for micro- and nanoelectronics technologies. Proc. SPIE, 7025. https://doi.org/10.1117/12.802356 (2008)

15. Varentsov, V.: Proposal for a new laser ablation ion source for LASPEC and MATS testing. https://doi.org/10.13140/RG.2.2.10904.39686

16. Varentsov, V., Günther, D., Hattendorf, B., Dietiker, R., Egorova, T.: Laser-ablation ion source with ion funnel: International application published under the patent cooperation treaty (pct). www.google.com/ patents/WO2012003946A1?cl=en (2012)

17. Querci, L., Varentsov, V., Günther, D., Hattendorf, B.: An rf-only ion funnel interface for ion cooling in laser ablation time of flight mass spectrometry. Spectrochim. Acta B At. Spectrosc. 146, 57 (2018). https://doi.org/10.1016/j.sab.2018.05.004

18. Brunner, T., Fudenberg, D., Sabourov, A., Varentsov, V.L., Gratta, G., Sinclair, D.: A setup for ba-ion extraction from high pressure Xe gas for double-beta decay studies with EXO. Nucl. Instrum. Methods Phys. Res., Sect. B 317, 473 (2013). https://doi.org/10.1016/j.nimb.2013.05.086

19. Brunner, T., Fudenberg, D., Varentsov, V., Sabourov, A., Gratta, G., Dilling, J., DeVoe, R., Sinclair, D., Fairbank, W., Albert, J.B., Auty, D.J., Barbeau, P.S., Beck, D., Benitez-Medina, C., Breidenbach, 
M., Cao, G.F., Chambers, C., Cleveland, B., Coon, M., Craycraft, A., Daniels, T., Daugherty, S.J., Didberidze, T., Dolinski, M.J., Dunford, M., Fabris, L., Farine, J., Feldmeier, W., Fierlinger, P., Gornea, R., Graham, K., Heffner, M., Hughes, M., Jewell, M., Jiang, X.S., Johnson, T.N., Johnston, S., Karelin, A., Kaufman, L.J., Killick, R., Koffas, T., Kravitz, S., Krücken, R., Kuchenkov, A., Kumar, K.S., Leonard, D.S., Leonard, F., Licciardi, C., Lin, Y.H., Ling, J., MacLellan, R., Marino, M.G., Mong, B., Moore, D., Odian, A., Ostrovskiy, I., Ouellet, C., Piepke, A., Pocar, A., Retiere, F., Rowson, P.C., Rozo, M.P., Schubert, A., Smith, E., Stekhanov, V., Tarka, M., Tolba, T., Tosi, D., Twelker, K., Vuilleumier, J.L., Walton, J., Walton, T., Weber, M., Wen, L.J., Wichoski, U., Yang, L., Yen, Y.R.: An RF-only ion-funnel for extraction from high-pressure gases. Int. J. Mass Spectrom. 379, 110 (2015). https://doi.org/10.1016/j.ijms.2015.01.003

20. Morrissey, D.J., Sherrill, B.M., Steiner, M., Stolz, A., Wiedenhoever, I.: Commissioning the A1900 projectile fragment separator. Nucl. Instrum. Methods Phys. Res., Sect. B 204, 90 (2003). https://doi.org/10.1016/S0168-583X(02)01895-5

21. Cooper, K., Sumithrarachchi, C.S., Morrissey, D.J., Levand, A., Rodriguez, J.A., Savard, G., Schwarz, S., Zabransky, B.: Extraction of thermalized projectile fragments from a large volume gas cell. Nucl. Instrum. Methods Phys. Res., Sect. A 763, 543 (2014). https://doi.org/10.1016/j.nima.2014.06.075

22. Barquest, B.R., Bollen, G., Mantica, P.F., Minamisono, K., Ringle, R., Schwarz, S., Sumithrarachchi, C.S.: Rfq beam cooler and buncher for collinear laser spectroscopy of rare isotopes. Nucl. Instrum. Methods Phys. Res., Sect. A 866, 18 (2017). https://doi.org/10.1016/j.nima.2017.05.036

23. Minamisono, K., Mantica, P.F., Klose, A., Vinnikova, S., Schneider, A., Johnson, B., Barquest, B.R.: Commissioning of the collinear laser spectroscopy system in the becola facility at nscl. Nucl. Instrum. Methods Phys. Res., Sect. A 709, 85 (2013). https://doi.org/10.1016/j.nima.2013.01.038

24. Krämer, J., König, K., Geppert, C., Imgram, P.B., Maaß, J., Meisner, E., Otten, W., Passon, S., Ratajczyk, T., Ullmann, J., Nörtershäuser, W.: High-voltage measurements on the 5 ppm relative uncertainty level with collinear laser spectroscopy. Metrologia 55(2), 268 (2018). https://doi.org/10.1088/1681-7575/aaabe0

25. Imgram, P., König, K., Krämer, J., Ratajczyk, T., Müller, R.A., Surzhykov, A., Nörtershäuser, W.: Collinear laser spectroscopy at ion-trap accuracy: Transition frequencies and isotope shifts in the $6 s^{2} S_{1 / 2} \rightarrow 6 p^{2} P_{1 / 2,3 / 2}$ transitions in $\mathrm{Ba}^{+}$. Phys. Rev. A 99(1). https://doi.org/10.1103/ PhysRevA.99.012511 (2019)

26. Imgram, P., König, K., Krämer, J., Ratajczyk, T., Maßß, B., Müller, P., Sommer, F., Nörtershäuser, W.: High-precision collinear laser spectroscopy at the collinear apparatus for laser spectroscopy and applied physics, PLATAN proceedings (2020)

27. Nörtershäuser, W., Campbell, P.: Laspec at fair's low energy beamline: A new perspective for laser spectroscopy of radioactive nuclei. Hyperfine Interactions 171(1-3), 149 (2006). https://doi.org/10.1007/s10751-006-9492-1

28. Varentsov, V.L., Ignatiev, A.A.: Numerical investigations of internal supersonic jet targets formation for storage rings. Nucl. Instrum. Methods Phys. Res., Sect. A 413(2-3), 447-456 (1998). https://doi.org/10. 1016/S0168-9002(98)00354-4

29. Heathcote, M.J. The J \& P Transformer Book: A Practical Technology of the Power Transformer, 13th edn. Elsevier and Newnes, Amsterdam [u.a.] and Oxford (2008)

Publisher's note Springer Nature remains neutral with regard to jurisdictional claims in published maps and institutional affiliations. 